
\title{
25 Research Sourere \\ Combining single-cell genomics and metagenomics to improve assembly in complex microbial communities
}

Koji Arikawa

Keigo Ide

Masato Kogawa

Tatsuya Saeki

Takuya Yoda

Taruho Endoh

Ayumi Matsuhashi

Haruko Takeyama

Masahito Hosokawa

\section{Video Byte}

Keywords: Microbiome, single-cell genomics, metagenomics, binning, software, genome assembly, de novo assembly, computational genomics, framework, microfluidics, microbial community, microbial ecology, genomics, analysis, techniques, bioinformatics, methods, human microbiome, microbiota

Posted Date: October 13th, 2021

DOI: https://doi.org/10.21203/rs.3.rs-967543/v1

License: (c) (i) This work is licensed under a Creative Commons Attribution 4.0 International License. Read Full License 


\section{Abstract}

High-quality reference genomes are needed to understand the physiology and function of uncultured microbes in complex ecosystems. Metagenomics has been an incredibly useful tool for studying microbial communities, but assigning sequence assemblies accurately to genomes is difficult in microbial species or strains that lack a reference genome. These 'consensus genomes' have lower resolution than those generated from cultured isolates. Combining single-cell genomics with metagenomics may allow us to overcome these methodological weaknesses. Thus, researchers recently developed a framework called SMAGLinker, which integrates single-cell genomes from microfluidic droplets and uses them as guides for metagenome assembly. Compared to metagenomics alone, SMAGLinker showed more precise contig binning and higher recovery rates of rRNA and plasmids in a mock microbial community. In human gut and skin microbiota samples, SMAGLinker returned more genomes than the conventional metagenomics frameworks. SMAGLinker was the only technique tested to identify that one human skin sample had two different strains of Staphylococcus hominis. The conventional metagenomics frameworks only returned one (chimeric) genome for S. hominis. This study demonstrates that SMAGLinker can return strain-resolved, high-quality draft genomes and suggests that it can be useful where reference genome expansion and strain-resolved analysis are needed. 